\title{
Behandlungskosten können den Therapieerfolg beeinflussen
}

Fragestellung: Wie wirkt sich der Preis einer medikamentösen Behandlung auf den Therapieerfolg aus?

Hintergrund: Daten aus experimentellen Untersuchungen und Befunde aus klinischen Studien belegen, dass der Therapieeffekt pharmakologischer Behandlungen durch die Kombination aus spezifischen pharmakologischen Effekten und Placeboeffekten bestimmt wird. Entscheidende Wirkvariablen, welche Placeboeffekten zugrunde liegen sind die Erwartungen und Vorerfahrungen des Patienten bezüglich einer Therapie. Diese Untersuchung ging der Frage nach, welchen Einfluss die (vermeintlichen) Kosten einer Behandlung auf den Placeboeffekt bei Morbus Parkinson hat.

Patienten und Methodik: In der kleinen aber kontrollierten Studie wurden zwölf Patienten im mittleren bis fortgeschrittenen Stadium der Parkinson-Krankheit im Rahmen eines Crossover-Designs zwei subkutane Kochsalzinjektionen verabreicht, welche die Studienteilnehmer für einen Dopaminagonisten hielten. Bei einer der zwei Injektionen wurden die Studienteilnehmer informiert, dass die Injektion 1.500 Dollar koste, bei der anderen, dass die Injektion

Espay AJ, Norris MN, Eliassen JC et al. Placebo effect of medication cost in Parkinson disease: A randomized double-blind study. Neurology 2015; 84: 794-802

\section{Dollar koste.}

Die Reihenfolge der teuren beziehungsweise günstigen Behandlung war randomisiert. Outcome-Parameter waren untersucherverblinde- te Ratings des UPDRS (Unified Parkinson Disease Rating Scale), des Purdue Pegboard Test sowie eines Finger Tapping Tasks. Außerdem wurden die zerebralen Blood-Oxygen-LevelDependent(BOLD)-Antworten während eines motorischen assoziativen Lernparadigmas mittels funktioneller Magnetresonanztomografie (fMRT) erfasst. Die Ergebnisse wurden mit den Verbesserungen unter L-Dopa verglichen, die vor der Randomisierung in die beiden Placeboarme im Rahmen eines On-OffVersuches ermittelt wurden.

Ergebnisse: Unter beiden Placebobehandlungen kam es zu einer signifikanten Verbesserung der Motorperformance in den drei Tests. Diese war allerdings signifikant stärker für das teure im Vergleich zu dem günstigen Placebo.

Nach Gabe des vermeintlich teuren Placebos kam es zu einer Verbesserung des UPDRS-III von $28 \%$, was sich nicht signifikant von der Verbesserung unter L-Dopa unterschied. Die Verbesserung des UPDRS-III nach dem vermeintlich günstigen Medikament betrug nur 13\%. Die Verbesserung der Motorik unter dem teuren Placebo war besonders groß, wenn die Studienteilnehmer dieses als erste Injektion erhielten.

Weiterhin berichten die Autoren unterschiedliche BOLD-Aktivierungen in sensomotorischen Hirnarealen zwischen den Gruppen.

Schlussfolgerungen: Die Kosten einer Behandlung können den Placeboeffekt und damit auch den gesamten Therapieeffekt einer medikamentösen Behandlung beeinflussen.

\section{- Kommentar von Ulrike Bingel, Essen}

\section{Kein Freifahrtschein für teure Therapien}

Diese sehr kleine Studie repliziert Studienergebnisse aus dem Bereich der Placeboanalgesie, wo schon vor einigen Jahren gezeigt werden konnte, dass vermeintlich teure Analgetika eine signifikant stärkere Schmerzlinderung induzierten, als vermeintlich günstige [1]. Die aktuelle Studie unterstreicht damit erneut den erheblichen Einfluss von Erwartungseffekten auf den Therapieerfolg sowie den modulierenden Einfluss, den die Kosten von Behandlungen darauf haben können. Die bildgebenden Befunde lassen bei der sehr niedrigen Fallzahl sowie ohne Bezug zu Verhaltensdaten in dem untersuchten Task allerdings keine Rückschlüsse auf die zugrunde liegenden Mechanismen dieses Phänomens zu. Solche Studienergebnisse dürfen aber keinesfalls als Argument für den Einsatz teurer Medikamente instrumentalisiert werden. Vielmehr ist es eine wesentliche ärztliche Aufgabe, die Erwartung der Patienten an eine Therapie durch gezielte und individuell angepasste Kommunikation selbst zu beeinflussen und den Patienten nicht reflexiven Schlüssen von den Kosten auf den Wert der Behandlung zu überlassen.
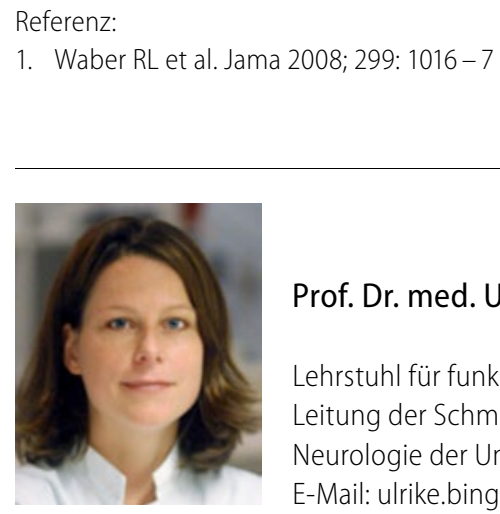

Prof. Dr. med. Ulrike Bingel, Essen

Lehrstuhl für funktionelle Bildgebung, Leitung der Schmerzambulanz, Klinik für Neurologie der Universitätsklinik Essen E-Mail: ulrike.bingel@uk-essen.de 